Pengju Huo, Xiaohong Li, Yang Liu and Haiying Qi*

\title{
Investigation on influences of loose gas on gas-solid flows in a circulating fluidized bed (CFB) reactor using full-loop numerical simulation
}

https://doi.org/10.1515/ijcre-2020-0119

Received July 26, 2020; accepted November 27, 2020;

published online December 4, 2020

\begin{abstract}
The influences of loose gas on gas-solid flows in a large-scale circulating fluidized bed (CFB) gasification reactor were investigated using full-loop numerical simulation. The two-fluid model was coupled with the QC-energy minimization in multi-scale theory (EMMS) gassolid drag model to simulate the fluidization in the CFB reactor. Effects of the loose gas flow rate, $Q$, on the solid mass circulation rate and the cyclone separation efficiency were analyzed. The study found different effects depending on $Q$ : First, the particles in the loop seal and the standpipe tended to become more densely packed with decreasing loose gas flow rate, leading to the reduction in the overall circulation rate. The minimum $Q$ that can affect the solid mass circulation rate is about $2.5 \%$ of the fluidized gas flow rate. Second, the sealing gas capability of the particles is enhanced as the loose gas flow rate decreases, which reduces the gas leakage into the cyclones and improves their separation efficiency. The best loose gas flow rates are equal to $2.5 \%$ of the fluidized gas flow rate at the various supply positions. In addition, the cyclone separation efficiency is correlated with the gas leakage to predict the separation efficiency during industrial operation.
\end{abstract}

Keywords: CFB reactor; cyclone separation efficiency; gas leakage; loose gas.

\section{Introduction}

The loose gas, which is also known as loose wind or aeration, is an important approach for controlling the

\footnotetext{
*Corresponding author: Professor Haiying Qi, Department of Energy and Power Engineering, Tsinghua University, Beijing 100084,

P. R. China, E-mail: hyqi@mail.tsinghua.edu.cn

Pengju Huo and Xiaohong Li, Hydrocarbon High-efficiency Utilization Technology Research Center, Shaanxi Yanchang Petroleum Co. Ltd, Xi'an 710075, P. R. China

Yang Liu, Key Laboratory for Thermal Science \& Power Engineering of Ministry of Education, Tsinghua University, Beijing 100084,

P. R. China
}

circulation and flow state of the solid particle materials inside the circulating fluidized bed (CFB) (Geng et al. 2015; Guan et al. 2016; Ma, Lei, and Xiao 2018; Wang et al. 2011). In view of the fact that the industrial-scale CFB gasification reactor is a complex full-loop system comprised of the riser, standpipe, multi-stage cyclones, loop seal, and other parts, the control of the loose gas flow rate, $Q$, is quite difficult. If $Q$ is too low in any part of the system (such as in the standpipe or loop seal), the particle circulation will be discontinuous with a low solid mass circulation rate, resulting in poor heat and mass transporting capability inside the bed. On the contrary, a large loose gas flow rate will lead to three problems. First, a high loose gas flow rate increases the possibility of gas leakage into the cyclones which reduces the separation efficiency (Huang et al. 2018a, 2018b). Second, it will cause type-B choking in the standpipe which leads to large pulsations in the CFB system (Bi, Grace, and Zhu 1993; Matsen 1982). Finally, too much nitrogen, which is commonly used as the loose gas in CFB gasification systems, will be mixed into the syngas, leading to the reduction in the reaction rates by diluting the other reactive gases (Liu et al. 2020b). However, in actual operations, the control of the loose gas is mostly based on experiences without sufficient theoretical analyses of the internal flow state. Therefore, numerical studies of the gas-solid fluidization throughout the fullloop CFB system are needed to predict the best supply rate of the loose gas.

Full-loop modeling of the gas-solid flows in the CFB reactor needs to accurately describe the various multi-phase flow structures in different parts (such as the riser, cyclone, and standpipe) of the reactor under variable fluidization conditions. However, it is well known that the fluidized gassolid flow is extremely non-uniform due to the strong particle clustering (Chen 2016). The particle clusters not only make various parameters fluctuate violently but also strongly affect the interactions between the gas and the solids, especially the gas-solid drag (Chen, Dai, and Qi 2016; Dai, Chen, and Qi 2015, 2016; Wang 2020). The existing numerical methods for industrial CFB simulation, whether the Eulerian-Eulerian method (i.e., two-fluid model) or the Eulerian-Lagrange method, still cannot reasonably and accurately account for the complex drag reduction caused 
by particle clusters (Chen 2016; Chen, Dai, and Qi 2016), which brings challenges to accurately model the fluidization process in the $\mathrm{CFB}$ reactor.

The gas-solid drag model, QC-EMMS, was developed to provide an effective drag modeling method based on the Energy Minimization in Multi-scale theory (EMMS) (Li and Kwauk 1994). The model includes two mathematical relations describing the feature parameters of the meso-scale clusters (i.e., the solid holdup and the cluster size) (Chen 2016). It predicts the gas-solid slip velocity, drag force reduction, heterogeneity degree of the flow, and the CFB operating parameters along with the singlepeak relations for various key variables as functions of the local solid volume fraction (Chen 2016). The QC-EMMS model can obtain the drag function for various conditions as has been verified by a series of experimental data (Chen, Dai, and Qi 2015). Therefore, the QC-EMMS model was applied in this study to calculate the gas-solid drag in the entire CFB reactor.

This study analyzes the effect of the loose gas on the gas-solid flow characteristics of a CFB gasification reactor to determine a reasonable loose gas flow rate, $Q$, for industrial operation. The study used the two-fluid model coupled with the QC-EMMS drag model to describe the fullloop CFB fluidization processes under various loose gas flow rates. $Q$ was gradually reduced in various loose gas supply regions to analyze the resulting variations of the solid mass circulation rate, gas leakage into the cyclones, and the cyclone separation efficiency, so as to determine the best loose gas flow rates in various locations.

\section{Schematic of the CFB reactor}

The simulated device was a $60 \mathrm{~m}$ high CFB coal gasification reactor (Li et al. 2020; Liu et al. 2020a, 2020b) as shown in Figure 1. In the field test condition, nitrogen was used as the fluidized gas that was injected uniformly from the riser bottom. A certain amount of loose gas $\mathrm{N}_{2}$ was also injected at three regions (L1 L3 marked with the red lines in Figure 1) located at the loop seal, the standpipe, and the return leg. The injection of loose gas was to return the particles back into the riser. The solid particles carried out of the riser by the fluidized gas first flowed into the 1st cyclone in various flow streams:

(1) The particles separated by the 1st cyclone drop into the loop seal and then return to the standpipe as they are driven by the loose gas injected at region L1.

(2) The particles not separated by the 1st cyclone enter the 2nd cyclone for further separation. The separated

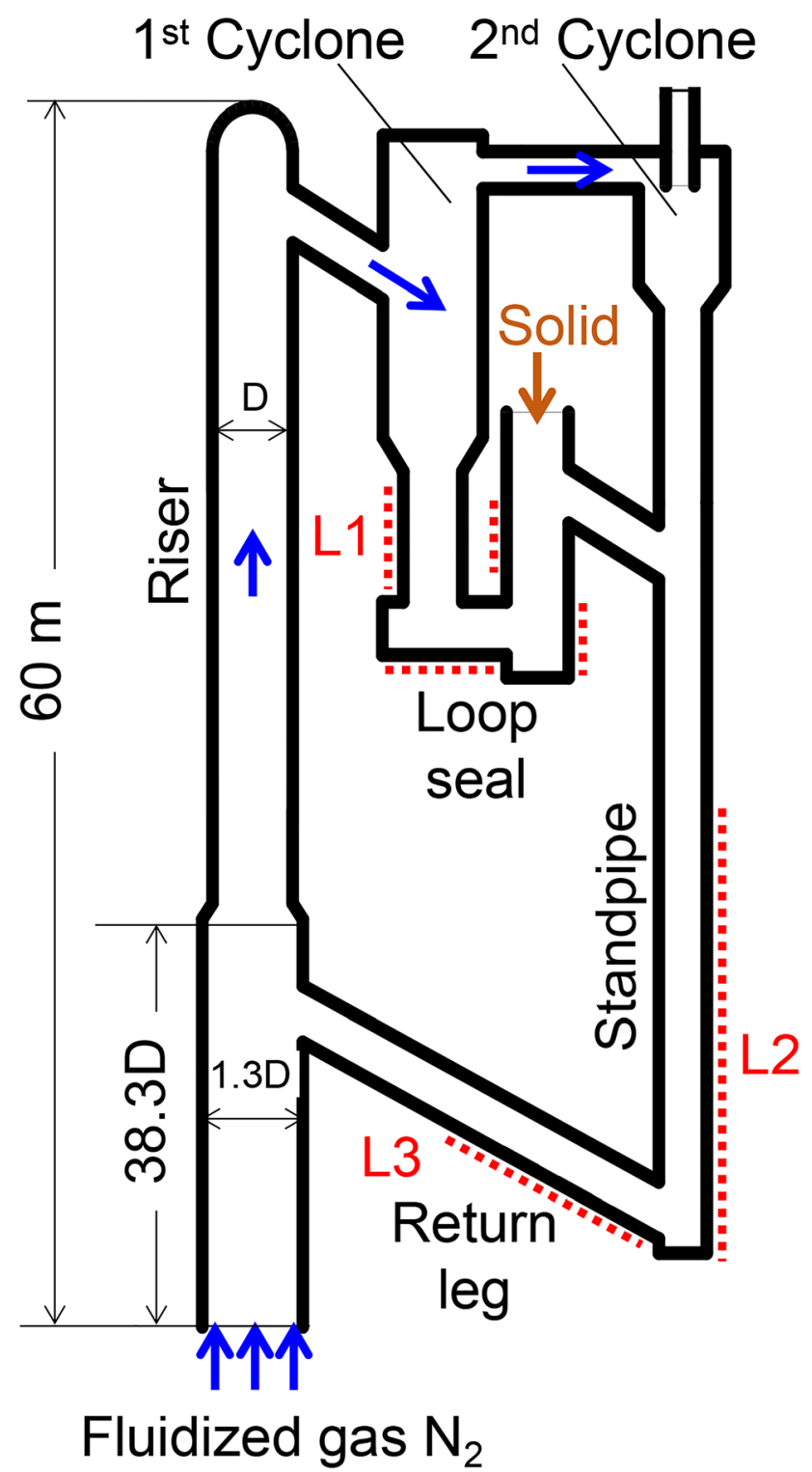

Figure 1: Geometry of the simulated circulating fluidized bed (CFB) reactor.

particles then drop into the standpipe while the other particles flow out of the CFB from the 2nd cyclone outlet.

(3) The particles in the standpipe are driven by the loose gas injected along region L2 to flow back to the riser through the return leg. A low flow rate of loose gas was also injected along region L3 to ensure the smooth return of the solid particles.

Since the CFB reactor contained three loose gas supply regions (L1 L3), the loose gas flow rate, $Q$, at each region not only affected the local gas-solid flow states but also influenced the gas flow direction and solid circulation rate in the whole system. However, the flow details inside the 
plant could not be analyzed in field tests due to measurement limitations. Therefore, numerical simulations were used to investigate the internal flow states for various loose gas flow rates to determine the best supply rate of the loose gas.

\section{Numerical models}

The commonly used two-fluid method (Benzarti et al. 2014; Liu et al. 2013) with the kinetic theory of granular flow was used in the particle flow simulations. The detailed model equations and solving method could be found in the previous work (Chen 2016). The drag function, $\beta$, in the momentum equations of the two-fluid model is the key to the simulation accuracy (Chen 2016). The drag function, $\beta$, for heterogeneous gas-solid flows need to be modified by a correction factor, $H_{d}$, (less than 1) to reflect the reduction of the homogeneous drag function, $\beta_{\text {homo }}$, due to particle clustering (Chen 2016). In this paper, $\beta_{\text {homo }}$ was calculated by the Wen-Yu model with the QC-EMMS model used to predict $H_{d}$ (Chen 2016; Chen, Dai, and Qi 2016).

$$
\begin{aligned}
\beta & =H_{d} \beta_{\text {hоmo }} \\
\beta_{\text {homo }} & =\frac{3}{4} C_{D 0} \frac{\varepsilon_{s} \varepsilon_{g} \rho_{g}\left|\vec{u}_{g}-\vec{u}_{s}\right|}{d_{p}} \varepsilon_{g}^{-2.65}, \\
C_{D 0} & =\left\{\begin{array}{ll}
\frac{24\left(1+0.15 \operatorname{Re}^{0.687}\right)}{\operatorname{Re}}, & \operatorname{Re}<1000 \\
0.44, & \operatorname{Re} \geq 1000
\end{array} .\right.
\end{aligned}
$$

\subsection{Drag calculation}

The QC-EMMS model, which was established based on EMMS theory (Li and Kwauk 1994), is a gas-solid drag model adaptive for non-uniform gas-solid fluidization (Chen 2016). The model can predict the drag reduction caused by particle clusters with the resulting drag function, $\beta$, agreeing well with the experimental results (O'Brien and Syamlal 1993) as shown in Figure 2.

The model includes two relations for the cluster solidholdup, $\varepsilon_{s c}$, and the cluster size, $d_{c}$, that are integrated into the EMMS theory to predict $H_{d}$ (Chen 2016). The heterogeneity factor, $\Psi$ that indicates the degree of the flow heterogeneity, in Eq. (3) is a function of different CFB operating parameters (Liu et al. 2020a, 2020b).

$$
\begin{gathered}
\varepsilon_{s c}=\varepsilon_{s}+\Psi \frac{211.74 \varepsilon_{s}^{2}}{e^{\left(3.098 \varepsilon_{s}+0.9398\right)^{2}}-2.42}\left(\varepsilon_{s, m f}-\varepsilon_{s}\right) \\
d_{c l}=\left[\frac{\varepsilon_{s c}-\varepsilon_{s}}{\varepsilon_{s, m f}-\varepsilon_{s}} \frac{\left[\frac{U_{p}}{1-\varepsilon_{\max }}-\left(U_{m f}+\frac{\varepsilon_{m f} U_{p}}{1-\varepsilon_{m f}}\right)\right] g}{N_{s t} \frac{\rho_{p}}{\rho_{p}-\rho_{g}}-\left(U_{m f}+\frac{\varepsilon_{m f} U_{p}}{1-\varepsilon_{m f}}\right) g}+1\right] d_{p}
\end{gathered}
$$

In this study, the drag correction factor, $H_{d}$, was developed for the field test conditions $\left(0.14 \mathrm{MPa}, 100^{\circ} \mathrm{C}\right)$ as shown in Table 1. $H_{d}$ is correlated with the local solid volume fraction, $\varepsilon_{s}$, and the gas-solid slip velocity, $u_{\text {slip }}$, as shown in Figure 3.

\subsection{Mesh and boundary conditions}

The unstructured tetrahedral mesh was used in the simulation. Fine elements were adopted at the loose gas injection ports in the three supply regions (L1 L3) as shown in Figure 4. To ensure grid independence, the grid size was no larger than 300 times of the particle diameter in Table 1 (Chen 2016; Liu et al. 2020b).

\begin{tabular}{|c|c|c|c|c|c|}
\hline \multirow[t]{2}{*}{ Parameters } & \multicolumn{2}{|r|}{ Particle } & \multicolumn{2}{|c|}{ Nitrogen } & \multirow[t]{2}{*}{ Superficial gas velocity $U_{g}, \mathrm{~m} / \mathrm{s}$} \\
\hline & Density, $\mathrm{kg} / \mathrm{m}^{3}$ & Sauter mean diameter, $\mu \mathrm{m}$ & Density, $\mathrm{kg} / \mathrm{m}^{3}$ & Viscosity, Pa.s & \\
\hline Values & 2565 & 90 & 1.26 & $2.1 \times 10^{-5}$ & 1.7 \\
\hline
\end{tabular}

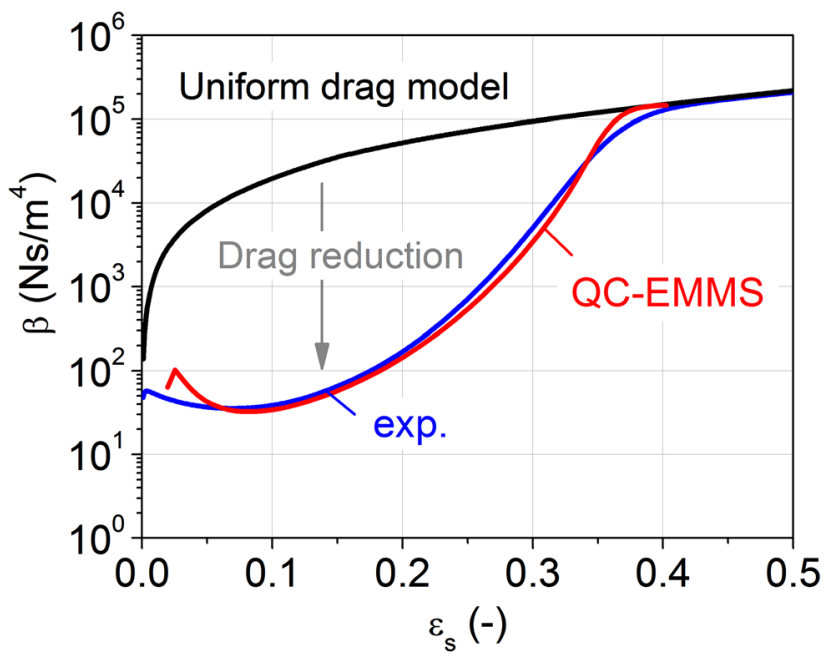

Figure 2: Drag reduction reproduced by the QC-EMMS model (Chen 2016; Li et al. 2020; Liu et al. 2020b).

Table 1: Conditions for calculating the drag correction factor. 


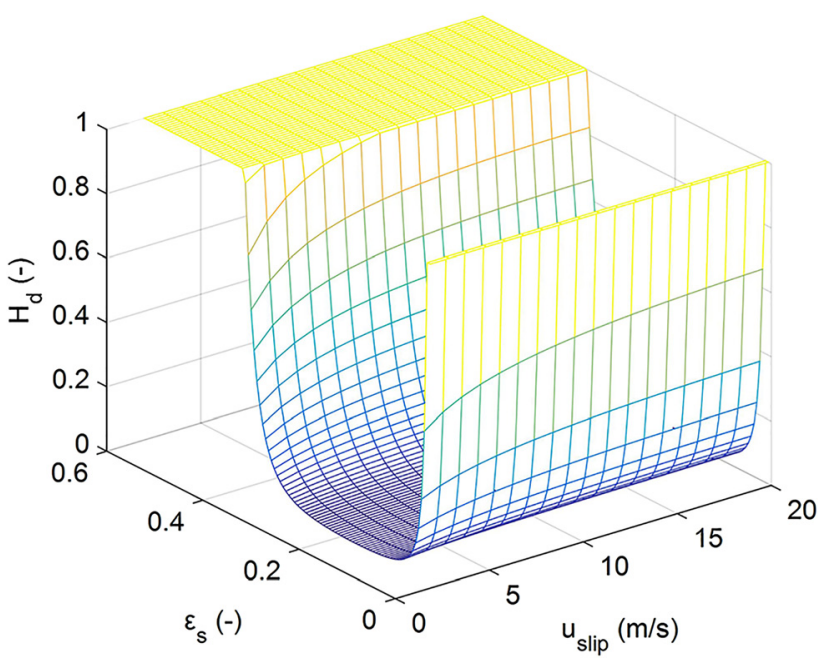

Figure 3: Drag correction factor used in the simulations.

Nitrogen was used as both the fluidized gas and the loose gas with sand used for the bed material. The gas and solid phase properties are listed in Table 1. The fluidized gas and loose gas inlets were all set as velocity inlets. The 2nd cyclone outlet was set as a pressure outlet with the pressure equal to 0.14 MPa. The non-slip condition was used for the gas phase along the walls, and partial-slip condition was used for the particulate phase according to the previous study (Benyahia 2012). The solid flow rate at the solid inlet (indicated by the brown arrow in Figure 1) was set equal to

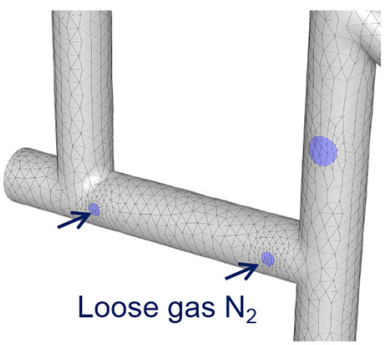

L1

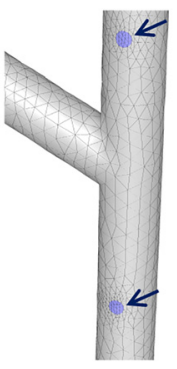

L2

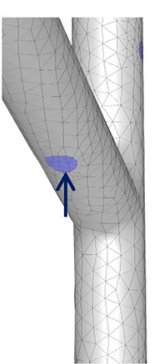

L3
Figure 4: Mesh structures at the various loose gas supply regions. that at the 2nd cyclone outlet through an user-defined function to ensure the solid material balance in the CFB.

\subsection{Loose gas flow rate settings}

The simulations were first conducted for the loose gas flow rate, $Q$, used in the field tests given as Case $\mathrm{T}$ in Table 2 to verify the simulation accuracy. $Q$ in regions $\mathrm{L} 1$ and $\mathrm{L} 2$ in Case $\mathrm{T}$ were both high loose gas ratios, $\zeta$, (i.e., the ratio of the loose gas flow rate to the fluidized gas flow rate) higher than $10 \%$.

Then, two groups of cases (A and B) were used to predict the influence of loose gas to find the best loose gas supply rates at various locations. Since that loose gas flow in region $\mathrm{L} 3$ was found to have little influence on the CFB flow state during the field test, only $Q$ in regions L1 and L2 were reduced in Cases A and B. Each group consisted of 6 settings of $Q$ with various loose gas ratios, $\zeta$.

Initially, the CFB contained a total sand mass of 4 tons. Some of the sand was uniformly distributed in the riser with the rest packed in the loop seal and the standpipe. Each simulation had a total time of $300 \mathrm{~s}$ with a time step of $2 \times 10^{-3} \mathrm{~s}$. The simulations were all carried out on the commercial simulation platform ANSYS Fluent, and the calculation time spend for each case was about 2 days.

\section{Results and discussion}

\subsection{Analysis of gas-solid flow states}

The predictions for the field test conditions, Case $\mathrm{T}$, are analyzed first to verify the simulation accuracy as well as to analyze the internal flow states.

\subsubsection{Riser pressure drop}

During the field tests, the pressure drop in the riser was measured by pressure probes. As shown in Figure 5, the simulated axial pressure drop, $\Delta p$, in riser agrees well with

Table 2: Loose gas flow rate settings.

\begin{tabular}{|c|c|c|c|c|c|c|c|c|c|c|c|c|c|}
\hline Case & $T^{*}$ & A1 & A2 & A3 & A4 & A5 & A6 & B1 & B2 & B3 & B4 & B5 & B6 \\
\hline$Q_{1}, \mathrm{Nm}^{3} / \mathrm{h}$ & 379.8 & 93 & 65 & 36 & 26 & 14 & 7.2 & \multicolumn{6}{|c|}{379.8} \\
\hline$\zeta_{1}, \%$ & 26.6 & 6.5 & 4.5 & 2.5 & 1.5 & 1 & 0.5 & \multicolumn{6}{|c|}{26.6} \\
\hline$Q_{2}, \mathrm{Nm}^{3} / \mathrm{h}$ & 169.8 & \multicolumn{6}{|c|}{169.8} & 93 & 65 & 36 & 22 & 14 & 7.2 \\
\hline$\zeta_{2}, \%$ & 11.8 & \multirow{2}{*}{\multicolumn{5}{|c|}{11.8}} & & 6.5 & 4.5 & 2.4 & 1.5 & 1 & 0.5 \\
\hline$Q_{3}, \mathrm{Nm}^{3} / \mathrm{h}$ & & & & & & & 105 & & & & & & \\
\hline
\end{tabular}

*Field test conditions. 


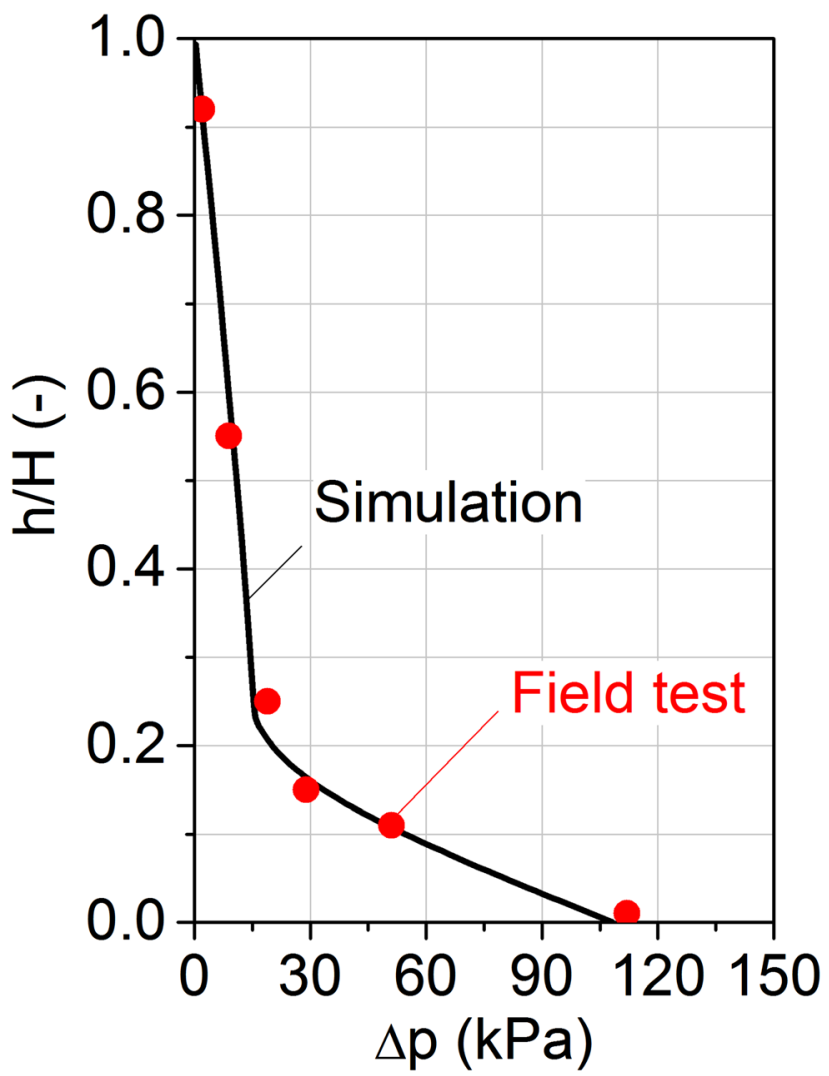

Figure 5: Pressure drop distribution along the height of the riser.

the field test data measured with relative errors less than $9 \%$. The low superficial gas velocity, $U_{g}$, of $1.7 \mathrm{~m} / \mathrm{s}$ causes the particles to accumulate at the riser bottom, resulting in the formation of a dense phase region and a large $\Delta p$. Above the dense phase region, the pressure drop is quite small due to the dilute particle distribution there. Axial pressure drop shows a clear division between bottomdense and upper-dilute regions.

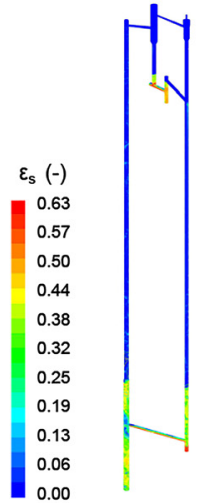

(A) Full loop

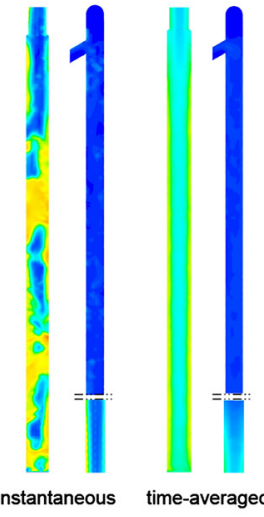

(B) Riser

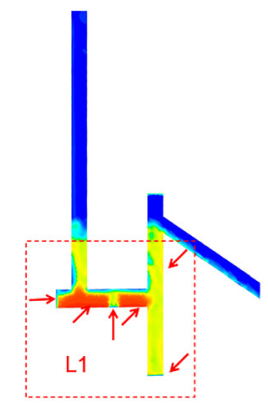

(C) Loop seal

\subsubsection{Solid volume fraction}

Consistent with the axial pressure drop distribution shown in Figure 5, the particles in the riser accumulate near the riser bottom. As can be seen from the instantaneous solid volume fraction, $\varepsilon_{s}$, distribution in Figure $6 \mathrm{~A}, \mathrm{~B}$, strong particulate fluctuations exists in the gas-solid flow field. The simulation reproduced the core-annular flows, which is the typical structure in the CFB chemical reactor, along the riser radius as seen in the time-averaged distributions (Weber et al. 2018).

The loose gas flow rates, $Q$, in region $\mathrm{L} 1$ at the loop seal and region $\mathrm{L} 2$ at the standpipe have loose gas flow ratios, $\zeta$, both larger than $10 \%$. The particles in the loop seal and in the standpipe are fluidized with solid volume fractions of about 0.4 as shown in Figure 6C, D. However, such strongly fluidized flows caused by the high $Q$ will reduce the gas sealing of the particles in the standpipe and loop seal, which may increase the gas leakage into the cyclones and reduce the cyclone separation efficiency (Huang et al. 2018a, 2018b). Therefore, additional studies are needed to evaluate the effectiveness of the loose gas supply rates in various locations, the degree of gas leakage, and the separation efficiencies of the two-stage cyclones in the CFB.

\subsubsection{Gas leakage and cyclone separation efficiency}

As shown in Table 3 and Figure 7, some of the loose gas leaks into the cyclones due to the ineffective solid particle seals in the standpipe and loop seal caused by the high loose gas flow rates:

(1) Since the L1 loose gas supply region is close to the two cyclones with the lowest pressure in the CFB, the loose gas supplied from region L1 all leaks into the two cyclones since the gas leakage ratio, $\delta$, (i.e., the ratio of

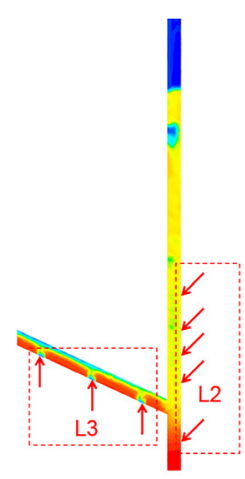

Figure 6: Distributions of the solid volume (D) Standpipe \& Return leg

fraction in different parts of the reactor. 
Table 3: Gas leakage from the three loose gas supply regions.

\begin{tabular}{lrrr}
\hline $\begin{array}{l}\text { Supply } \\
\text { region }\end{array}$ & $\begin{array}{r}\text { Gas leakage into } \\
\text { the } 1 \text { st cyclone, } \\
\boldsymbol{q}_{\mathbf{1}}, \mathbf{N m}^{3} / \mathbf{h}\end{array}$ & $\begin{array}{r}\text { Gas leakage into } \\
\text { the } 2 \text { nd cyclone, } \\
\boldsymbol{q}_{\mathbf{2}}, \mathbf{N m}^{3} / \mathbf{h}\end{array}$ & $\begin{array}{r}\text { Gas leakage } \\
\text { ratio, } \boldsymbol{\delta}, \%\end{array}$ \\
\hline $\mathrm{L} 1$ & 191.4 & 188.4 & 100 \\
$\mathrm{~L} 2$ & 0 & 81.8 & 48.2 \\
$\mathrm{~L} 3$ & 0 & 0 & 0 \\
\hline
\end{tabular}

the gas leakage flow rate into the cyclones to the total supplied loose gas flow rate) is equal to $100 \%$. About $50 \%$ of the loose gas from L1 leaks into the 1st cyclone while the rest flows into the standpipe and then leaks into the 2nd cyclone.

(2) $48.2 \%$ of the loose gas supplied from region L2 leaked into the 2nd cyclone through the standpipe with the rest flowing into the riser through the return leg due to being entrained with the circulating particles.

(3) The loose gas from region L3 all directly flows into the riser through the return leg without leakage.

The results show that the gas leakage problems in the CFB are serious. The gas leakage flow rate into the 1st cyclone, $q_{1}$, is $191.4 \mathrm{Nm}^{3} / \mathrm{h}$ while that into the 2 nd cyclone, $q_{2}$, is even higher with a flow rate of $270.2 \mathrm{Nm}^{3} / \mathrm{h}$ (summing the flow rates of gas leakage from L1 and L2, $q_{2,1}$ and $q_{2,2}$ ). The gas leakage causes the upward gas flow at the bottom of the two cyclones with positive axial velocities, $u_{\text {axis }}$, as shown in Figure 8, which reduces the swirling intensity and then the effective separation space in the cyclones (Huang et al. 2018b). Therefore, the separation efficiencies, $\eta$, of the 1 st and 2nd cyclone are only 83 and $78 \%$, which are lower than the design efficiencies of over $90 \%$.

The field test measurements verify the simulation accuracy when using the QC-EMMS model. The simulation results show the gas leakage and low cyclone separation efficiency problems caused by the excessive loose gas flow rate, $Q$. Therefore, further simulations were performed with lower $Q$ in Case A and B (Table 2) to investigate the effects of loose gas flow rates, so as to determine the best loose gas supply rate in various locations.

\subsection{Influences of the loose gas flow rate}

\subsubsection{Particle flow patterns}

Figure 9 shows the influence of the loose gas flow rate from region L1 (loop seal) on the particle flow patterns. As the loose gas ratio in region $\mathrm{L} 1, \zeta_{1}$, decreases, the friction resistance between particles in the loop seal increases, leading to the denser solid distribution and the higher height of the particle seal as shown in Figure 9A. Then, the reduction of the particle return rate from the loop seal to the standpipe reduces the height of the particle seal in the standpipe with decreasing $\zeta_{1}$ as shown in Figure 9B. This then reduces the particle return rate from the standpipe to the riser, which slows down the particle circulation in the entire CFB.

As shown in Figure 10, reducing the loose gas ratio in region $\mathrm{L} 2, \zeta_{2}$, increases the solid volume fraction in the standpipe and reduces the height of the particle seal. However, when $\zeta_{2}$ is too low, the particle seal height in the

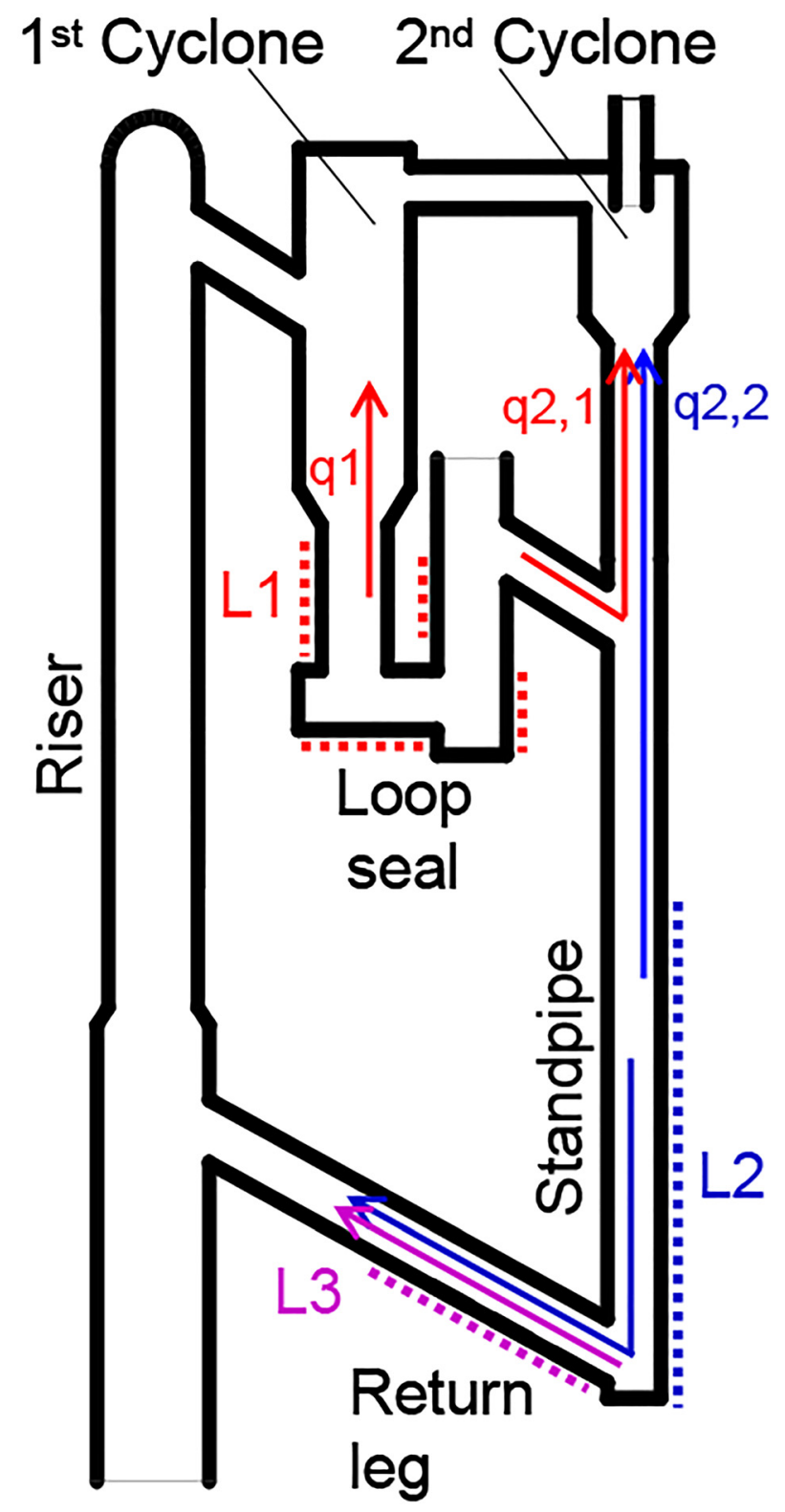

Figure 7: Schematic diagram of the loose gas flow directions. 


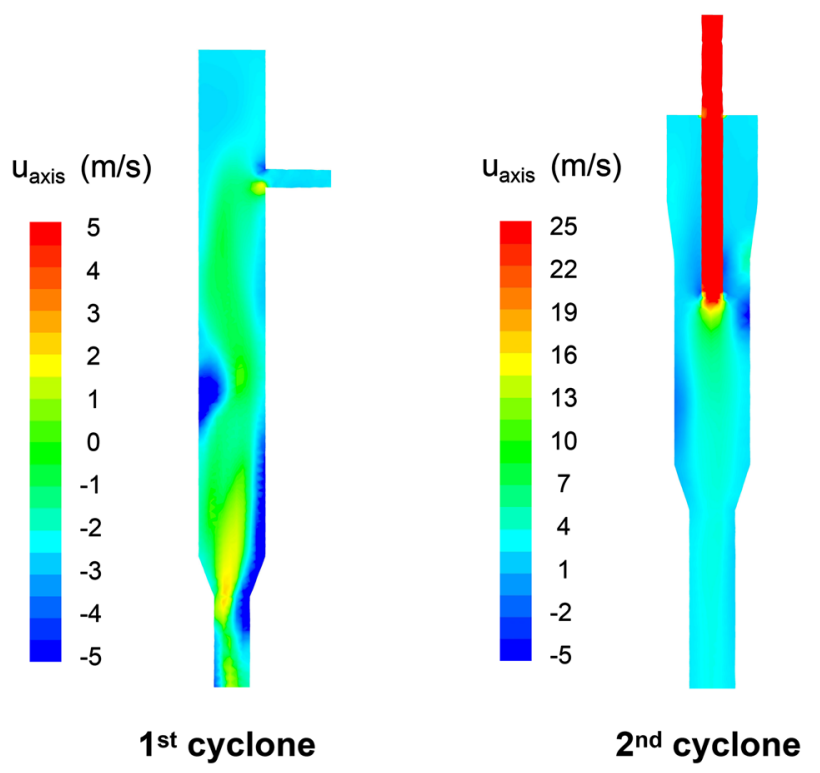

Figure 8: Axial velocity distributions in the two cyclones. standpipe increases due to the reduction of the particle return rate from the standpipe to the riser under high particle friction. The variation of $\zeta_{2}$ has no influence on the flow state in the loop seal.

\subsubsection{Solid mass circulation rate}

As shown in Figure 11, the solid mass circulation rate, Gs, has similar variations with $\zeta_{1}$ and $\zeta_{2}$ :

(1) When $\zeta_{1}$ or $\zeta_{2}$ is over $2.5 \%$, Gs does not change with the loose gas flow rate. This means that the particle driving capability of the loose gas has reached saturation and Gs in the CFB has reached a maximum for the existing superficial gas velocity, $U_{g}$.

(2) When $\zeta_{1}$ or $\zeta_{2}$ is less than $2.5 \%$, the loose gas flow rate significantly affects the solid mass circulation rate. As the loose gas ratio decreases, the flow resistances of the particles in the loop seal and standpipe increase

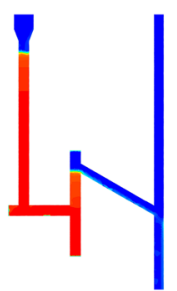

(a) Loop seal
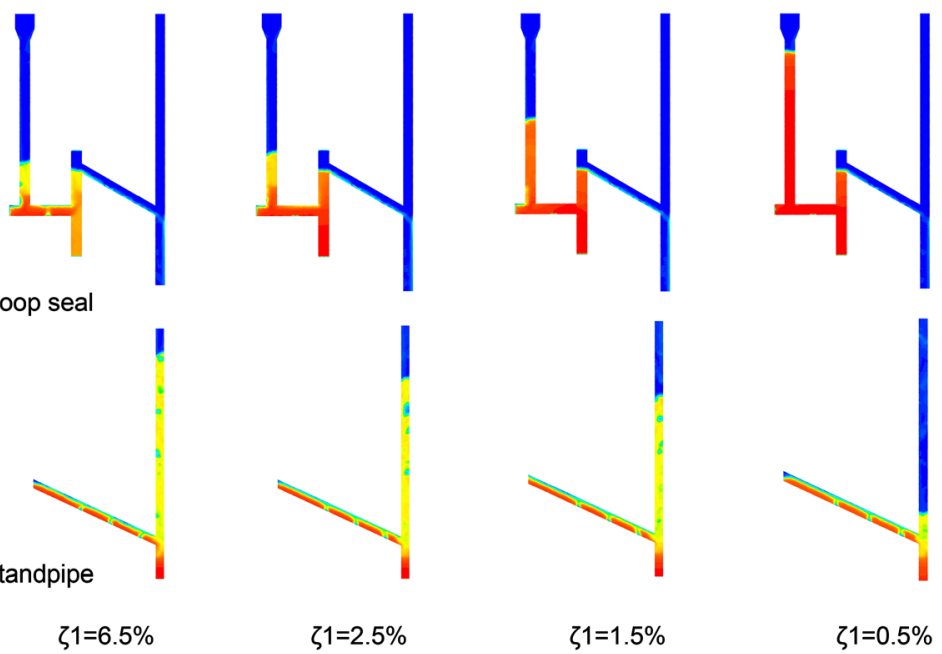

Figure 9: Solid volume fraction distributions (b) Standpip

$\zeta 1=6.5 \%$

$\zeta 1=2.5 \%$

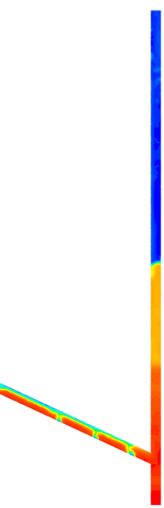

$\zeta 2=1.5 \%$

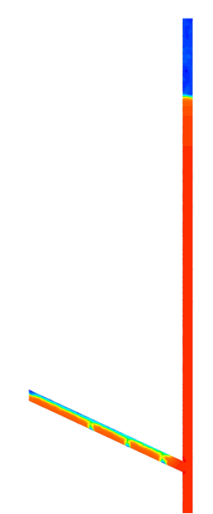

Figure 10: Solid volume fraction distributions for various loose gas ratios, $\zeta_{2}$ $\zeta 2=0.5 \% \quad$ (Case B). 


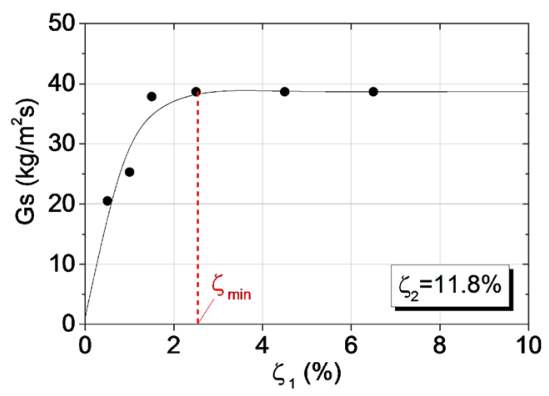

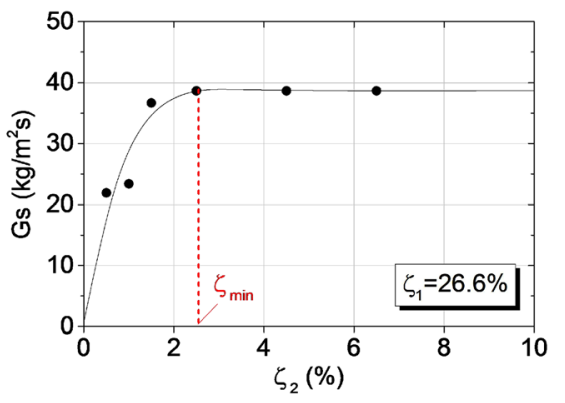

Figure 11: Variations of the solid mass circulation rate with the loose gas ratios at $\mathrm{L} 1$ and $\mathrm{L} 2$.
(Section 4.2.1), which reduces the particle flow rate returning from the standpipe to the riser and then the overall Gs.

Thus, the simulated minimum loose gas ratio, $\zeta_{\min }$, to maximize $G s$ is $2.5 \%$. Larger loose gas flow rates cannot increase the particle circulation in the CFB but will increase the gas leakage into the cyclones which reduces the separation efficiency as noted in Section 4.1.3. It should be noted that the similar variation of $G s$ with loose gas flow rate are also reported in some studies by using full-loop circulating fluidized bed simulation (Geng et al. 2015; Guan et al. 2016; Lee et al. 2014). However, due to the differences in CFB reactor structure, particle properties, and operating conditions, the values of $\zeta_{\min }$ in various studies are different from each other and also inconsistent with the value of $2.5 \%$ in this work.

\subsubsection{Cyclone separation efficiency}

Reducing the loose gas flow ratio, $\zeta$, causes particles to accumulate more tightly in the loop seal or standpipe (Section 4.2.1) which more effectively seals the gas flow. Therefore, the gas leakage rates into the 1 st cyclone, $q_{1}$, and into the 2nd cyclone, $q_{2}$, (sum of $q_{2,1}$ from region L1 and $q_{2,2}$ from $L 2$ ) are both significantly reduced with decreasing $\zeta$ as shown in Figure 12.

Analysis of the simulated cyclone separator efficiency, $\eta$, for all the simulation cases showed that $\eta$ decreases linearly with increasing relative gas leakage rates, $q^{\star}$, (i.e., the ratio of the gas leakage rate, $q$, to the fluidized gas flow rate) as shown in Figure 13. The predicted trend is consistent with the experimental and simulation results from Huang et al. (2018a, 2018b). The swirling intensity and the tangential gas velocity in the cyclones become lower with the increasing gas leakage. Then, more particles inside the cyclones cannot be effectively separated but flow out of the CFB reactor directly. Figure 13 shows that increasing $q^{\star}$ by 0.05 reduces the separation efficiency by
$3 \%$. The separation efficiency exceeds $90 \%$ with no gas leakage $\left(q^{\star}=0\right)$.

It can be concluded that reducing the loose gas flow rate improves the cyclone separation efficiency. However, an excessively small loose gas flow rate will reduce the

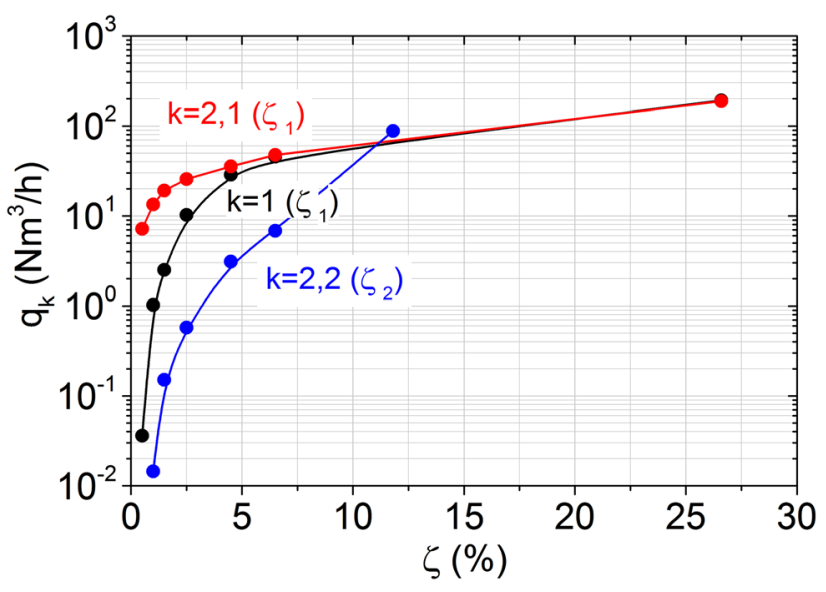

Figure 12: Gas leakage rates into the cyclones for various loose gas ratios.

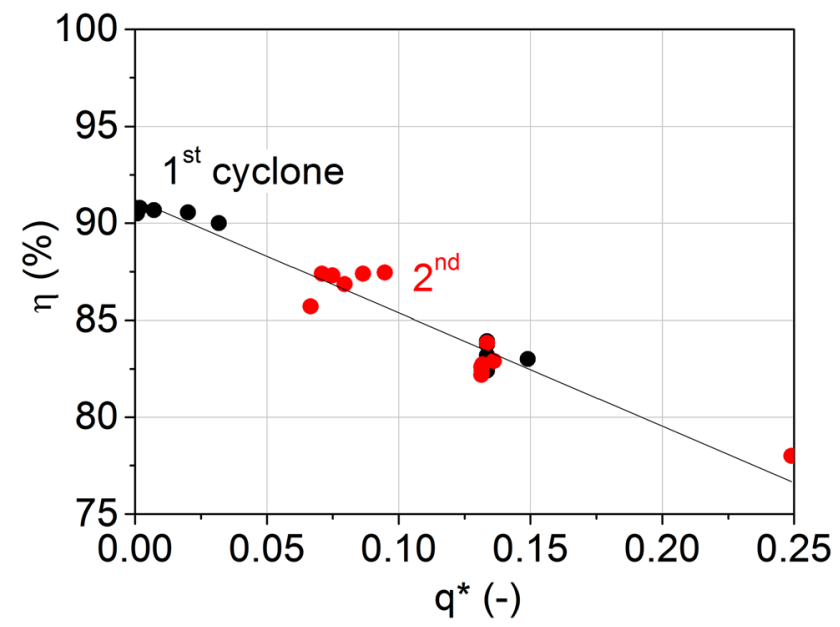

Figure 13: Cyclone separator efficiency for various gas leakage flow rates. 
circulation rate in the CFB (Section 4.2.2). For these reasons, the best loose gas supply is to set the loose gas ratios in regions $\mathrm{L} 1$ and $\mathrm{L} 2$ both to $2.5 \%$ (i.e., the minimum loose gas ratio, $\zeta_{\min }$, that maximizes $G s$ ) so as to ensure the highest cyclone separation efficiency for the highest particle circulation rate in the CFB.

\subsubsection{Correlation for the cyclone separation efficiency}

The cyclone separation efficiency, $\eta$, of the CFB plant cannot be measured in the field, so a correlation is needed for $\eta$ based on the predicted results for the cyclone separation efficiency during industrial operation. The Lapple formula was used to calculate the separation efficiency, $\eta_{0}$, without gas leakage into the cyclone (Lapple 1950):

$$
\begin{aligned}
& \eta_{0}=\frac{1}{1+\left(d_{50} / d_{p}\right)^{m}}, \quad d_{50}=\sqrt{\frac{9 \mu_{g} w}{2 \pi \rho_{p} V_{\text {in }} N}}, \\
& N=1.68 \ln \left(1+0.566 V_{\text {in }}\right)
\end{aligned}
$$

In Equation (5), $d_{50}$ is the particle diameter with a separation efficiency of $50 \%, w$ and $V_{\text {in }}$ are the inlet port width and the inlet gas velocity into the cyclone, and $N$ is the effective spiral number for separation. $m$ is an empirical coefficient related to the cyclone structure obtained by correlating the predicted separation efficiency for gas leakage rates less than $10 \mathrm{Nm}^{3} / \mathrm{h}$. The correlation gave $m$ equal to 1.21 with fitting errors less than $2 \%$.

The proportionality between the gas leakage flow rate and the loose gas ratio shown in Figure 12 for the cyclone design shown in Figure 7 was used to modify the separation efficiency given by Eq. (5) to predict the separation efficiency reduction caused by gas leakage shown in Figure 13:

$$
\eta=\eta_{0}\left(1-k \zeta^{0.3}\right), \quad k=0.01 \times\left(-0.5 V_{\text {in }}+19.93\right)
$$

When Eq. (6) is used for the 1st cyclone of the CFB, $\zeta$ is taken as the loose gas ratio in the loop seal, $\zeta_{1}$. For the 2nd cyclone, the calculation uses the total loose gas ratio from the loop seal and the standpipe (i.e., $\zeta_{1}+\zeta_{2}$ ). The loose gas ratios and the other parameters can be substituted into Eqs. (5) and (6) to predict the current cyclone separation efficiency during industrial operations.

\section{Conclusions}

(1) The gas-solid flows inside the full-loop CFB reactor were studied using numerical simulations. A minimum loose gas ratio, $\zeta_{\min }$ that maximizes the solid mass circulation rate is about $2.5 \%$. Thus, a further increase of the loose gas flow rate over $\zeta_{\min }$ only aggravates the gas leakage into cyclones and reduces the cyclone separation efficiency. Therefore, the best loose gas supply is setting the loose gas ratios near $\zeta_{\text {min }}$.

(2) A correlation was developed for the cyclone separation efficiency as a function of the gas leakage. The correlation can be used in industrial applications to predict cyclone separation performance.

Author contributions: All the authors have accepted responsibility for the entire content of this submitted manuscript and approved submission.

Research funding: This work was supported by Shaanxi Yanchang Petroleum Co. Ltd.

Conflict of interest statement: The authors declare no

\begin{tabular}{|c|c|}
\hline$C_{D O}$ & single particle drag coefficient \\
\hline$d_{c l}$ & particle cluster size $\mu \mathrm{m}$ \\
\hline$d_{p}$ & single particle size $\mu \mathrm{m}$ \\
\hline$d_{50}$ & $\begin{array}{l}\text { single particle size for the cyclone separation efficiency of } \\
50 \% \mu \mathrm{m}\end{array}$ \\
\hline$D$ & diameter m \\
\hline$g$ & gravitational acceleration $\mathrm{m} / \mathrm{s}^{2}$ \\
\hline Gs & solid mass circulation rate $\mathrm{kg} / \mathrm{m}^{2} \mathrm{~s}$ \\
\hline$h$ & local riser height $\mathrm{m}$ \\
\hline$H$ & total riser height $\mathrm{m}$ \\
\hline$H_{d}$ & drag correction factor \\
\hline$m$ & empirical coefficient related to the cyclone structure \\
\hline$N$ & effective spiral number for separation \\
\hline$N_{s t}$ & $\begin{array}{l}\text { mass-specific energy consumption for suspending particles } \\
\mathrm{J} / \mathrm{kg}\end{array}$ \\
\hline$q$ & gas leakage rate into the cyclones $\mathrm{Nm}^{3} / \mathrm{h}$ \\
\hline$q^{*}$ & relative gas leakage rate into the cyclones \\
\hline$Q$ & loose gas flow rate $\mathrm{Nm}^{3} / \mathrm{h}$ \\
\hline $\operatorname{Re}$ & particle Reynolds number \\
\hline$u$ & local velocity $\mathrm{m} / \mathrm{s}$ \\
\hline$u_{\text {axis }}$ & local axial velocity $\mathrm{m} / \mathrm{s}$ \\
\hline$u_{\text {slip }}$ & local gas-solid slip velocity $\mathrm{m} / \mathrm{s}$ \\
\hline$U_{g}$ & superficial gas velocity $\mathrm{m} / \mathrm{s}$ \\
\hline$U_{m f}$ & minimum fluidization velocity $\mathrm{m} / \mathrm{s}$ \\
\hline$U_{p}$ & superficial solid velocity $\mathrm{m} / \mathrm{s}$ \\
\hline$V_{\text {in }}$ & cyclone inlet gas velocity $\mathrm{m} / \mathrm{s}$ \\
\hline$w$ & cyclone inlet port width $\mathrm{m}$ \\
\hline
\end{tabular}
conflicts of interest regarding this article.

\section{Nomenclature}

\section{Greek letters}

$\begin{array}{ll}\beta & \text { heterogeneous drag function } \mathrm{Ns} / \mathrm{m}^{4} \\ \beta_{\text {homo }} & \text { homogeneous drag function } \mathrm{Ns} / \mathrm{m}^{4} \\ \delta & \text { gas leakage ratio } \% \\ \Delta p & \text { pressure drop } \mathrm{kPa} \\ \varepsilon & \text { volume fraction } \\ \varepsilon_{\max } & \text { maximum gas volume fraction }\end{array}$


$\varepsilon_{m f} \quad$ minimal fluidization gas volume fraction

$\varepsilon_{s, m f} \quad$ minimal fluidization solid volume fraction

$\varepsilon_{s c} \quad$ solid hold-up in the clusters

$\zeta \quad$ loose gas ratio \%

$\zeta_{\min }$ minimum loose gas ratio to maximize solid mass circulation rate $\%$

$\eta \quad$ cyclone separation efficiency $\%$

$\eta_{0} \quad$ cyclone separation efficiency without gas leakage \%

$\mu_{g} \quad$ gas viscosity Pa.s

$\rho \quad$ density $\mathrm{kg} / \mathrm{m}^{3}$

$\Psi \quad$ heterogeneity factor

\section{Subscripts}

$g \quad$ gas phase

$s, p \quad$ solid phase

\section{References}

Benyahia, S. 2012. "Analysis of Model Parameters Affecting the Pressure Profile in a Circulating Fluidized Bed." AIChE Journal 58 (2): 427-39.

Benzarti, S., H. Mhiri, H. Bournot, and R. Occelli. 2014. "Numerical Simulation of Turbulent Fluidized Bed with Geldart B Particles." Advanced Powder Technology 25 (6): 1737-47.

Bi, H. T., J. R. Grace, and J. X. Zhu. 1993. "Types of Choking in Vertical Pneumatic Systems." International Journal of Multiphase Flow 19 (6): 1077-92.

Chen, C. 2016. Investigation on Mesoscale Structure in Gas-Solid Fluidization and Heterogeneous Drag Model. Berlin: Springer Press.

Chen, C., Q. Dai, and H. Qi. 2016. "Improvement of EMMS Drag Model for Heterogeneous Gas-Solid Flows Based on Cluster Modeling." Chemical Engineering Science 141: 8-16.

Dai, Q., C. Chen, and H. Qi. 2015. “A Generalized Drag Law for Heterogeneous Gas-Solid Flows in Fluidized Beds.” Powder Technology 283: 120-27.

Dai, Q., C. Chen, and H. Qi. 2016. "Influence of Meso-Scale Structures on Drag in Gas-Solid Fluidized Beds." Powder Technology 288: 87-95.

Geng, C., W. Zhong, Y. Shao, D. Chen, and B. Jin. 2015. “Computational Study of Solid Circulation in Chemical-Looping Combustion Reactor Model." Powder Technology 276: 144-55.

Guan, Y., J. Chang, K. Zhang, B. Wang, Q. Sun, and D. Wen. 2016. “ThreeDimensional Full Loop Simulation of Solids Circulation in an Interconnected Fluidized Bed.” Powder Technology 289: 118-25.

Huang, Y., M. Zhang, J. Lyu, Z. Liu, and H. Yang. 2018a. "Effects of Gas Leakage on the Separation Performance of a Cyclone. Part 1:
Experimental Investigation." Chemical Engineering Research and Design 136: 900-05.

Huang, Y., M. Zhang, J. Lyu, Z. Liu, and H. Yang. 2018b. "Effects of Gas Leakage on the Separation Performance of a Cyclone. Part 2: Simulation." Chemical Engineering Research and Design 136: 906-15.

Lapple, C. E. 1950. "Gravity and Centrifugal Separation.” American Industrial Hygiene Association Quarterly 11: 40-7.

Lee, D., M. W. Seo, T. D. B. Nguyen, W. C. Cho, and S. D. Kim. 2014. "Solid Circulation Characteristics of the Three-Reactor ChemicalLooping Process for Hydrogen Production." International Journal of Hydrogen Energy 39 (27): 14546-56.

Li, J., and M. Kwauk. 1994. Particle-Fluid Two-Phase Flow, the Energy Minimization Multi-Scale Method. Beijing: Metallurgical Industry Press.

Li, X., P. Huo, Y. Liu, and H. Qi. 2020. "Numerical Study of Load Characteristics of a CFB Coal Gasifier by Using the Generalized Drag Model." Energy \& Fuels 34 (4): 5084-94.

Liu, G., P. Wang, S. Wang, L. Sun, Y. Yang, and P. Xu. 2013. “Numerical Simulation of Flow Behavior of Liquid and Particles in LiquidSolid Risers with Multi Scale Interfacial Drag Method." Advanced Powder Technology 24: 537-48.

Liu, Y., P. Huo, X. Li, and H. Qi. 2020a. "Numerical Analysis of the Operating Characteristics of a Large-Scale CFB CoalGasification Reactor with the QC-EMMS Drag Model.” Canadian Journal of Chemical Engineering, https://doi.org/10.1002/cjce. 23911 (in press).

Liu, Y., P. Huo, X. Li, and H. Qi. 2020b. "Numerical Study of Coal Gasification in a Dual-CFB Plant Based on the Generalized Drag Model QC-EMMS." Fuel Processing Technology 203: 106363.

Ma, Q., F. Lei, and Y. Xiao. 2018. “Numerical Analysis of Operating Conditions for Establishing High-Density Circulating Fluidized Bed by CPFD Method." Powder Technology 338: 446-57.

Matsen, J. M. 1982. "Mechanisms of Choking and Entrainment." Powder Technology 32 (1): 21-33.

O'Brien, T., and M. Syamlal. 1993. "Particle Cluster Effects in the Numerical Simulation of a Circulating Fluidized Bed." Preprint Volume for CFB-IV 430-35.

Wang, J. 2020. "Continuum Theory for Dense Gas-Solid Flow: A State-Of-The-Art Review." Chemical Engineering Science 215: 115428.

Wang, S., G. Liu, H. Lu, J. Chen, Y. He, and J. Wang. 2011. “Fluid Dynamic Simulation in a Chemical Looping Combustion with Two Interconnected Fluidized Beds." Fuel Processing Technology 92 (3): 385-93.

Weber, J. M., M. M. Bobek, R. W. Breault, J. S. Mei, and L. J. Shadle. 2018. "Investigation of Core-Annular Flow in an Industrial Scale Circulating Fluidized Bed Riser with Electrical Capacitance Volume Tomography (ECVT)." Powder Technology 327: 524-35. 